\title{
Understanding the ecological fallacy
}

In an Endgames article by Philip Sedgwick "Understanding the ecological fallacy” (BMJ 2015;351:h4773, doi:10.1136/bmj. h4773) the definition of Spearman's correlation coefficient was incorrect. Answer c) should read: Spearman's rank correlation coefficient measured the strength of a monotonic association between trimethoprim prescription and antibiotic resistance across practices. Statement c remains "true." Under the heading
"Answers," the text should read: The data were analysed using Spearman's rank correlation coefficient, 3 which measured the strength of the monotonic association between rates of trimethoprim prescription and the proportion of patients with urine samples identified with trimethoprim resistant Gram negative bacteria across the 28 general practices (c is true). 